\title{
Formación de Estudiantes Tutores: Un Apoyo Fundamental para Potenciar el Desarrollo de Prácticas de Física
}

\author{
Alicia A. Bravo (1), Claudio A. Faúndez (1), Felipe A. Moraga ${ }^{(2)}$ y María A. Borzone ${ }^{(2)}$. \\ (1) Depto. De Física, Facultad de Ciencias Físicas y Matemáticas, Universidad de Concepción, Barrio \\ Universitario s/n, Casilla 160-C, Concepción, Chile (e-mail: aliciabravo@udec.cl, claudiofaundez@udec.cl). \\ (2) Centro de Apoyo al Desarrollo de Estudiantes, Universidad de Concepción, Barrio Universitario s/n, Casilla \\ 160-C, Concepción, Chile (e-mail: femoraga@udec.cl, mborzonev@udec.cl).
}

Recibido Jun. 1, 2018; Aceptado Ago. 3, 2018; Versión final Oct. 4, 2018, Publicado Abr. 2019

\begin{abstract}
Resumen
Este artículo presenta un estudio de caso que describe la implementación de una asignatura complementaria que está orientada a generar herramientas, prácticas y actitudinales, que contribuyan a mejorar el desempeño docente de estudiantes tutores en el área de la física universitaria. En el transcurso de la asignatura se observa el logro de competencias metodológicas propias de la didáctica y de una visión constructivista del aprendizaje, herramientas necesarias en la tutoría y ayudantías, puesto que permiten el desarrollo y la aplicación de habilidades del pensamiento. En la asignatura se efectuaron diversas actividades con el fin potenciar una futura realización de prácticas de física. Los resultados fueron favorables puesto que los estudiantes aprobaron la asignatura con buenas calificaciones y mostraron una mejoría en su actitud discursiva y una mayor confianza en sí mismos, además se encuentran satisfechos con lo aprendido. Estos resultados propician el desarrollo de prácticas que favorezcan el aprendizaje significativo en estudiantes que cursen asignaturas de física en la Universidad de Concepción, Chile.
\end{abstract}

\section{Formation of Teaching Assistants: A Fundamental support for Enhancing the Development of Physics Assistantship}

\begin{abstract}
This article presents a case study that describes the implementation of a complementary course, oriented to generate practical and attitudinal tools that contribute to improving the performance of teaching assistants in the area of university physics. As the course advances, the achievement of methodological competences, proper of didactics, and a constructive view of learning are observed. These are necessary tools in tutoring and assistantships, since they allow the development and application of cognitive skills. During the course, several activities were carried out with the aim of enhancing the future development of assistantships in physics. Results were favorable since students approved the course with overall good marks and showed an enhancement in their discursive abilities and greater self-trust. Moreover, students claim to be satisfied. These results encourage the development of assistantships that favor significant learning in students that undergo physics courses at the University of Concepción, Chile
\end{abstract}

Keywords: teaching assistants, academic formation; university teaching 


\section{INTRODUCCIÓN}

Es imprescindible reconocer la importancia que tiene para las universidades la formación de capital humano, no sólo como constituyente estructural sino también como uno de los principales factores de cambio; y al mismo tiempo, el más fuerte estabilizador de esos cambios cuando se instala en la cultura institucional (Garrido, 2007). La Organización para la Cooperación y el Desarrollo Económico (OCDE) plantea que, tanto las universidades como otras instituciones de educación superior, juegan un papel trascendental en los sistemas de desarrollo de capital humano (OCDE, 2009:4). De aquí es que surge la preocupación de las universidades chilenas por los resultados de sus estudiantes. La reprobación, el rezago y el abandono de los estudiantes han impulsado la proposición de una serie de estrategias que buscan dar solución a los nuevos desafíos educacionales (Álvarez y González, 2005; Aguilar et al., 2017).

Algunos planes a modo de atención a estas problemáticas son: i) la preparación de nuevos profesionales que se sumen a las plantas docentes universitarias, ii) perfeccionamiento e instrucción en el área de didáctica de académicos que imparten clases y iii) perfeccionamiento de estudiantes que ejercen docencia en las ayudantías de ciertas asignaturas. Dentro de sus respectivas comunidades universitarias, hacen llamados públicos con el fin de tomar conciencia del propio quehacer educacional de los principales actores: docentes y estudiantes. Junto a estos llamados establecen ciertas metas y promueven los contenidos a enseñar para mejorar su labor educativa (Ver tabla 1), haciendo énfasis en la preparación didáctica y en el área psicológica educacional (Gairín et al., 2004). En nuestro país ya se han implementado programas de desarrollo docente, por lo general en forma de tallares o diplomados, que se caracterizan por temas como: la planificación de clases, el diseño de cursos, la evaluación al servicio del aprendizaje, didáctica, las metodologías activas y el uso de TICs (Espejo y González, 2014, Cabrera-Murcia, 2017). En la actualidad, la mayoría de las universidades alrededor del mundo tienen incorporado a su sistema educativo universitario iniciativas específicas pensadas para la formación de los nuevos profesores y todos aquellos docentes que deseen actualizarse (Monereo, 2013).

Tabla 1: Descripción de estrategias para enfrentar los desafíos en educación de algunas universidades chilenas.

\begin{tabular}{|c|c|c|}
\hline Universidad & Objetivos & Cursos \\
\hline $\begin{array}{l}\text { Pontificia } \\
\text { Universidad } \\
\text { Católica de } \\
\text { Valparaíso } \\
\text { (PUCV) }\end{array}$ & $\begin{array}{l}\text { Desarrollar competencias de apoyo a la docencia en los } \\
\text { ayudantes de la PUCV, de manera que los jóvenes } \\
\text { puedan fortalecer su colaboración en el logro de mejores } \\
\text { aprendizajes en los estudiantes de la asignatura } \\
\text { correspondiente. }\end{array}$ & $\begin{array}{l}\text { Nuevos Desafíos en Educación Superior. } \\
\text { Rol y ética que Caracteriza al Ayudante } \\
\text { PUCV. } \\
\text { Estrategias de enseñanza y aprendizaje. } \\
\text { Tecnologías para el Aprendizaje. } \\
\text { Evaluación para el Aprendizaje. } \\
\text { Comunicación Efectiva y Manejo de } \\
\text { Grupo. }\end{array}$ \\
\hline $\begin{array}{l}\text { Universidad } \\
\text { Arturo Prat } \\
\text { (UNAP) }\end{array}$ & $\begin{array}{l}\text { Desarrollar las características y rol de un ayudante, el } \\
\text { cual debiera tener "una actitud de entrega, compromiso, } \\
\text { generosidad y de interés por aprender formando parte } \\
\text { de un grupo interdisciplinario. Como también, es } \\
\text { fundamental que tengan altas expectativas de } \\
\text { desarrollo, posean liderazgo, sepan trabajar en equipo, } \\
\text { tengan habilidades comunicacionales, asertividad, entre } \\
\text { otras. }\end{array}$ & $\begin{array}{l}\text { Estilos de aprendizaje. } \\
\text { El modelo educativo UNAP. } \\
\text { Las metodologías activo-participativas. } \\
\text { El aprendizaje centrado en el estudiante. } \\
\text { La preparación de ayudantías. }\end{array}$ \\
\hline $\begin{array}{l}\text { Universidad } \\
\text { de Chile }\end{array}$ & $\begin{array}{l}\text { Establecer nuevos propósitos formativos sintonizados } \\
\text { con las demandas de la sociedad, la revisión de } \\
\text { metodologías de trabajo y nuevas formas de } \\
\text { participación de los actores involucrados en la relación } \\
\text { del enseñar y el aprender. }\end{array}$ & $\begin{array}{l}\text { Contexto, rol y buenas prácticas. } \\
\text { Aprendizaje y planificación. } \\
\text { Metodologías activas. } \\
\text { Evaluación y retroalimentación. } \\
\text { Habilidades interpersonales para la } \\
\text { docencia. }\end{array}$ \\
\hline $\begin{array}{l}\text { Universidad } \\
\text { Andrés Bello } \\
\text { (UNAB) }\end{array}$ & $\begin{array}{l}\text { Contar con ayudantes que desarrollen las competencias } \\
\text { necesarias para un proceso de docencia universitaria de } \\
\text { calidad, dando apoyo efectivo a los requerimientos de } \\
\text { los académicos y estudiantes. }\end{array}$ & $\begin{array}{l}\text { Expresión oral. } \\
\text { Didáctica. } \\
\text { Evaluación. } \\
\text { Tecnología educativa. }\end{array}$ \\
\hline $\begin{array}{l}\text { Universidad } \\
\text { de Santiago } \\
\text { de } \quad \text { Chile } \\
\text { (USACH) }\end{array}$ & $\begin{array}{l}\text { Mejorar su desempeño como ayudantes con un manejo } \\
\text { de conocimientos en dichas materias y colaborar en el } \\
\text { proceso formativo de los futuros arquitectos de la } \\
\text { Universidad. }\end{array}$ & $\begin{array}{l}\text { Herramientas en los campos de la } \\
\text { didáctica, evaluación y comunicación. }\end{array}$ \\
\hline
\end{tabular}

En el marco universitario, sin duda se ha marcado un antes y un después en el cuestionamiento de cómo y qué enseñar e, inevitablemente, de las formas de aprender, asunto que trae consigo la reflexión y toma de decisiones sobre distintos aspectos, como por ejemplo: la preparación y formación de los docentes universitarios, las bases metodológicas y evaluativas, la creación de estrategias y programas remediales, 
entre otros (Gairín et al., 2004; Monereo, 2013; Aguilar et al., 2017). Ahora, se está abriendo la posibilidad de instaurar poco a poco el constructivismo, modelo en el cual el estudiante pasa a ser el eje del proceso de enseñanza-aprendizaje, estableciendo así una formación orientada por competencias (Gairín et al., 2004; Álvarez y González, 2005; Adán et al., 2015). Esto conlleva a un cambio de metodologías, puesto que ha de considerarse, para la presentación de los contenidos, la diversidad de estudiantes en el aula, y junto con ello, los diferentes estilos de aprendizaje. Llevar a cabo esta metodología implica que toda la institución universitaria se convierta al paradigma constructivista, sobre todo profesores y estudiantes. Si las universidades desean responder a los desafíos actuales respecto de la calidad de la educación universitaria, no pueden seguir confiando en imitar figuras docentes que basen su didáctica en la exposición oral de contenidos, a la espera de que sean repetidos por estudiantes dispuestos sólo a seguir reproduciendo dichas materias (Monereo, 2013). En este contexto de cambio, la tutoría ha de jugar un papel relevante como función de acompañamiento personal y académico, en el desarrollo de habilidades y actitudes para el estudio, en el seguimiento y apoyo en las experiencias de aprendizaje del estudiante y en su construcción personal y profesional, dejando atrás su concepción tradicional de espacio, destinado a resolver dudas surgidas a la hora de estudiar y para ser un instrumento de orientación, gestión y personalización del aprendizaje (García et al., 2005; Arbizu at al., 2005; Narro y Arredondo, 2013; Adán et al., 2015; Solaguren-Beascoa y Moreno, 2016; Aguilar et al., 2017). En países anglosajones, se han realizado esfuerzos por introducir la figura del tutor universitario, con un rol relevante en la construcción de los aprendizajes (Colvin, 2007; Bulte et al., 2009; Baeten y Simons, 2014; Philipp et al, 2016a, 2016b; Baeten et al., 2018)

Se suma a este nuevo desafío la variabilidad de estudiantes que hoy ingresan a la universidad. En los últimos 30 años, en Chile, ha avanzado en la diversificación de la matrícula (Canales y De los Ríos, 2009) y la cobertura de la educación superior ha ido en aumento cada año, pero los números de ingreso no se condicen con los de egreso (SIES, 2014) (ver figura 1). Esta diferencia se produce por diversas razones; una de las más comunes es la alta tasa de deserción en los primeros años de universidad debido a factores académicos e institucionales; esto relacionado con la falta de integración de los estudiantes en las misma instituciones (Canales y de los Ríos, 2018). Debido a lo anterior es que las universidades buscan diferentes caminos para potenciar la integración y permanencia de los estudiantes, utilizándose para esto diferentes estrategias didácticas. Una de las más difundidas en el contexto de la docencia universitaria es la acción tutorial, entendida como una acción pedagógica y social destinada a implementar actividades que favorezcan el proceso de aprendizaje de los estudiantes y disminuir probabilidades de abandono (Baggini y del Valle, 2017). Este tipo de intervenciones se considera positiva por brindar acompañamiento académico y retroalimentación directa y personalizada (Tinto, 2010; Vega et al., 2014), siendo una estrategia recomendada en los primeros años como forma de facilitar la adaptación y aprendizaje de los estudiantes noveles (Graffigna et al., 2014).

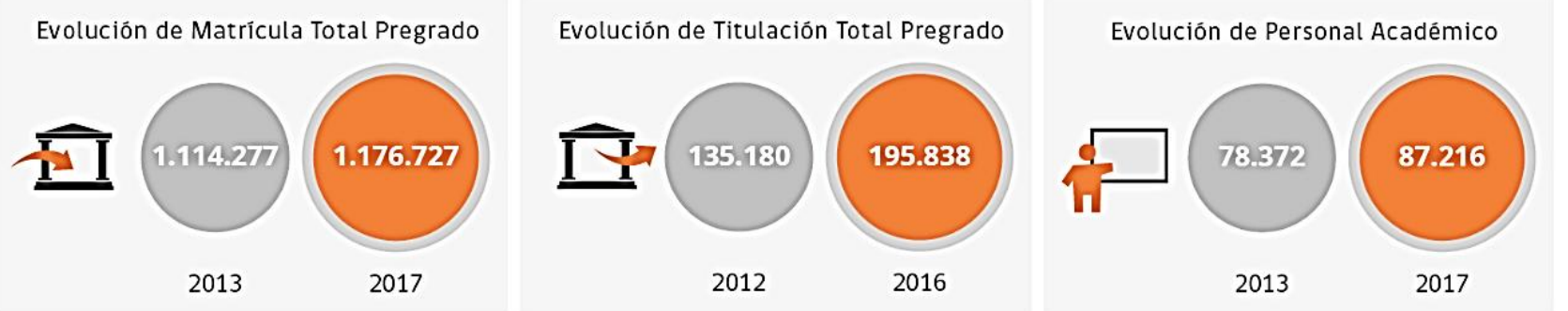

Fig. 1: Relación de ingreso y egreso de universidades chilenas (Tomado de: SIES 2014)

Si bien el principal responsable en la entrega de los conocimientos y el facilitador de su aprendizaje son los docentes, las horas teóricas no son suficientes para que el estudiante pueda analizar e interactuar con lo que le es nuevo, por lo menos en el área de las ciencias. Por lo tanto, se añaden las horas de práctica que según la asignatura contempla laboratorios prácticos 0 , en su mayoría y aún más comunes, las prácticas de desarrollo de ejercicios propuestos. Estas prácticas suelen estar a cargo de estudiantes de cursos superiores, los cuales deben realizar clases complementarias a las impartidas por el docente a cargo, reforzar y revisar contenidos, ser mediadores en la comunicación entre docentes y estudiantes y ejecutar y presentar la resolución de ejercicios que permiten acercar más al estudiante a los nuevos contenidos presentados. Pues bien, desde este punto de vista estos estudiantes llamados tutores pasan a ejercer un rol de educador, debiendo poseer casi las mismas competencias que se les exigen a los académicos universitarios, debido a esto es que los programas de formación de ayudantes se consideran como el primer paso de la carrera docente (García et al., 2012; Sommariva et al., 2013; Adán et al., 2015; Cabrera-Murcia, 2017).

En relación a las características que comparten tutores se encuentra el carácter informal de los entornos de trabajo, esto debido a la obvia falta de profesionalización de los estudiantes ayudantes, de hecho es esto lo que impide confundir el papel de los estudiantes tutores con la actividad docente del profesor. No tienen cualificación profesional, ni un papel evaluador formal, tampoco control sobre el currículo ni sobre los 
materiales utilizados (Falchikov, 2001; Menéndez, 2010). La falta de preparación profesional es el hecho que propicia una dinámica única en el aula, puesto que el tutor comparte la condición de estudiante universitario. Ejerce su máximo potencial por el hecho de que él mismo es un estudiante, se presenta como tal y, en consecuencia, se apresta a poner a disposición de sus compañeros su experiencia positiva sobre cómo afrontar aquellos aspectos problemáticos, que con casi total seguridad son los que afectan también a los estudiantes que en ese momento cursan la asignatura (Menéndez, 2010). Otra característica de la labor del tutor se refiere a la relación que se mantiene con los estudiantes que, aunque es asimétrica, es más simétrica que la que los estudiantes mantienen con el docente responsable de la asignatura. El tutor tiene la experiencia de enseñar como un docente pero a la vez la de ser estudiante; por esto es que las tutorías son consideradas una forma de fortalecer la docencia universitaria, ya que en este nivel educativo, el estudiante tutor ya ha demostrado tener habilidades y recursos para lograr una formación profesional. (Menéndez, 2010; García et al., 2012; Narro y Arredondo, 2013; Aguilar et al., 2017).

El entorno y las características propias de la labor del tutor se convierte en un recurso de importancia que complementa las prácticas habituales de aprendizaje, principalmente porque se aprovecha la zona de desarrollo próximo donde los estudiantes más aventajados ayudan a compañeros menos aventajados para aprender en el marco de un trabajo cooperativo, favoreciendo así una mejor comprensión, asimilación de la materia y un mejor rendimiento académico (Filtz y Gurung, 2012; McCavit y Zellner, 2016; Corona et al., 2018). Además se benefician los tutores, ya que adquieren competencias propias de la disciplina, mayor seguridad y habilidades comunicativas (Vygotsky, 1979; Bulte et al., 2009; Menéndez, 2010; Narro y Arredondo, 2013; Philipp, Tretter y Rick, 2016a, 2016b). En cuanto a las competencias necesarias a desarrollar en los tutores, suelen abordarse desde dos puntos de vista diferentes: (a) el saber hacer, desde esta perspectiva las competencias se entienden como capacidades, es decir, como un sistema especializado de recursos cognitivos y conocimientos necesarios para realizar un desempeño (Pérez et al., 2013); (b) una segunda perspectiva integradora, abarca competencia-desempeño; se entiende que el carácter complejo de las competencias se refiere al hecho de que los dominios no constituyen una habilidad muy específica; sino que suponen la movilización de recursos tanto individuales como del entorno para su adquisición y realización (Sospedra et al., 2013).

Las tutorías surgen como una estrategia con la que se pretende apoyar y asesorar a los estudiantes en su trayectoria universitaria y durante su proceso de adaptación, desarrollo y formación. Igualmente, pretende potenciar las capacidades de cada estudiante y ayudarles a superar las dificultades que van apareciendo con el fin de que éstos consoliden su proyecto académico con éxito (Álvarez y González, 2008; Badiali y Titus, 2010; Adán et al., 2015; Aguilar et al., 2017; Cabrera-Murcia, 2017; Corona et al., 2018). Para la ejecución de una acción tutorial se requiere conocer a los estudiantes: sus concepciones previas, lo que pueden aprender, los niveles de motivación, hábitos, actitudes y valores frente al estudio. Sommariva et al. (2013) indican que los tutores no son educadores profesionales, pero que son individuos que se dedican a la enseñanza y a ayudar a terceras personas en forma sistemática y propositiva. Sin embargo, estos autores señalan que para que exista efectividad en la intervención, un tutor debe ser guiado, estructurado y monitoreado. La eficacia del asesoramiento curricular y didáctico aumenta significativamente cuando el asesor es de la misma área de conocimiento que el tutor asesorado y ha recibido una formación específica en estrategias didácticas y de apoyo a colegas (Sánchez y Mayor, 2006; Cobos et al., 2018). Sommariva et al. (2013), respecto a la evaluación del programa colaborador académico- estudiantes, menciona que éste es de mucha ayuda, ya que aumenta el rendimiento y desempeño de los estudiantes. Más aún, los estudiantes indican que este tipo de programas fortalecen las relaciones interpersonales separando la amistad de sus roles de coeducadores.

El modelo universitario actual exige la formación de la acción tutorial, reformar a los docentes que por tradición han acabado asumiendo las tutorías como un simple formalismo vinculado a la transmisión del conocimiento o de preparación de su docencia, e impulsar a los estudiantes para que tengan un nuevo entendimiento de las tutorías, comprendiendo que estas no son sólo un servicio que la universidad les ofrece para ver los resultados académicos de los exámenes o preguntar dudas en relación a los mismos (Rumbo y Gómez, 2011). Los puntos planteados anteriormente y las problemáticas que se presentan en los primeros años de universidad, han evidenciado una alta tasa de reprobación en ramos fundamentales de ciencias y matemáticas, concentrando aquí la mayor cantidad de horas de ayudantías. En consecuencia, enfocándonos en un área en particular como es el proceso de enseñanza-aprendizaje de la física, donde se muestra un nivel de complejidad para los estudiantes, hemos determinado que se requiere la incorporación de metodologías innovadoras y herramientas cognitivas que, en conjunto con las tradicionales, logren mejores enseñanzas y adquisición de aprendizajes de esta disciplina en los estudiantes de pregrado. Para este desafío se requiere contar con estudiantes preparados en enseñanza cooperativa, resolución de problemas, metodologías didácticas y activas, y uso de entornos virtuales de aprendizaje. Es por ello que en este trabajo se presenta un curso de preparación para futuros tutores, el cual se caracteriza por ser teórico-participativo, entregando las competencias mínimas necesarias para que los estudiantes de Pedagogía en ciencias naturales y física, Licenciatura en física y carreras afín, puedan convertirse en tutores de los estudiantes inscritos en algún curso de física fundamental. 


\section{METODOLOGÍA}

La metodología de este estudio es de tipo cualitativa, se llevó a cabo un estudio de caso de tipo descriptivo (Yin, 1989). El objetivo fue describir la implementación de un curso complementario de formación de tutores. En primera instancia se presenta la modalidad y estrategias utilizadas en el curso y posteriormente se presentan resultados de las calificaciones obtenidas por los participantes y también los resultados de la encuesta de satisfacción con la asignatura que fue aplicada a los mismos participantes al finalizar el curso. Los participantes de estudio fueron 48 estudiantes inscritos en el complementario en el periodo 2015-2017. El curso de formación de tutores surge del Centro de Apoyo al Desarrollo del Estudiante (CADE), de la Universidad de Concepción (UdeC), debido a la necesidad de formalizar y dotar de estrategias necesarias a los estudiantes para que se desempeñan como tutores de las asignaturas de física fundamental. Las clases se basan en una metodología constructivista.

Los docentes vinculados a la preparación de los tutores fueron escogidos acorde a los módulos de enseñanza, quedando la planta docente compuesta por profesionales con postgrado en educación, psicología y física. Particularmente, este curso está orientado a estudiantes desde segundo año de carreras afines a física pertenecientes a la UdeC. Se impartió en la modalidad de asignatura complementaria, con el único prerrequisito de haber aprobado los cursos de físicas fundamentales. La tabla 2 muestra los resultados de aprendizaje esperados por tema del curso complementario.

Tabla 2: Resultados de aprendizaje de temáticas vistas en el curso.

\begin{tabular}{|c|c|}
\hline Tema & Resultados de Aprendizaje \\
\hline Aprendizaje significativo. & $\begin{array}{l}\text { 1. Explorar las formas en que los nuevos conocimientos y habilidades son estabilizados } \\
\text { en nuestra memoria en base a los postulados del aprendizaje significativo. } \\
\text { 2. Comprender los postulados del aprendizaje significativo. }\end{array}$ \\
\hline $\begin{array}{l}\text { Fundamentos de la } \\
\text { comunicación efectiva. }\end{array}$ & $\begin{array}{l}\text { 1. Identificar factores que influyen en el proceso enseñanza-aprendizaje. } \\
\text { 2. Conocer y clasificar los ruidos comunicativos que pueden presentarse en el desarrollo } \\
\text { de una ayudantía. } \\
\text { 3. Proponer estrategias para el control o prevención de ruidos comunicativos en el } \\
\text { desarrollo de una ayudantía. } \\
\text { 4. Reflexionar sobre factores y ruidos que pueden surgir en el desarrollo del proceso } \\
\text { enseñanza- aprendizaje de una ayudantía. }\end{array}$ \\
\hline $\begin{array}{l}\text { Teoría del aprendizaje de } \\
\text { Vigotsky y Aprendizaje } \\
\text { Cooperativo. }\end{array}$ & $\begin{array}{l}\text { 1. Conocer los postulados de la teoría del aprendizaje de Vigotsky. } \\
\text { 2. Reconocer aplicaciones de los postulados de la teoría del aprendizaje. } \\
\text { 3. Fundamentar la necesidad de trabajar de forma cooperativa en cada sesión. } \\
\text { 4. Reconocer el trabajo cooperativo como facilitador en procesos de andamiaje entre los } \\
\text { propios estudiantes. }\end{array}$ \\
\hline $\begin{array}{l}\text { Estrategias de resolución } \\
\text { de problemas en física. }\end{array}$ & $\begin{array}{l}\text { 1. Conocer los pasos a seguir para el correcto desarrollo de problemas de física. } \\
\text { 2. Reconocer que la estructuración mediante pasos facilita la obtención de resultados de } \\
\text { problemas planteados. }\end{array}$ \\
\hline $\begin{array}{l}\text { Aplicación del uso de } \\
\text { EVA. }\end{array}$ & $\begin{array}{l}\text { 1. Conocer en qué consisten los entornos virtuales de aprendizaje (EVA) } \\
\text { 2. Identificar la utilidad de los Eva en el proceso enseñanza-aprendizaje, mediante la } \\
\text { observación de aplicaciones de animaciones, simulaciones, software educativos, etc. }\end{array}$ \\
\hline $\begin{array}{l}\text { Uso del Aprendizaje } \\
\text { basado en problemas } \\
(\mathrm{ABP}) \text {. }\end{array}$ & $\begin{array}{l}\text { 1. Conocer la metodología de aprendizaje basado en problemas (ABP) } \\
\text { 2. Aplicar soluciones a problemas de física planteados mediante la técnica de aprendizaje } \\
\text { basado en problemas. }\end{array}$ \\
\hline $\begin{array}{l}\text { Uso de laboratorios } \\
\text { experimentales básicos. }\end{array}$ & $\begin{array}{l}\text { 1. Realizar laboratorios de bajo costo para apoyar a la comprensión de conceptos físicos } \\
\text { en las ayudantías } \\
\text { 2. Visualizar experimentalmente contenidos a tratar en las ayudantías }\end{array}$ \\
\hline
\end{tabular}

En la etapa de diagnóstico de necesidades se realizó una grabación de una exposición individual de la realización de una ayudantía de física. Esta exposición fue evaluada por los docentes y utilizada como pre test.

En la etapa de aplicación de la asignatura complementaria, se utilizó un diseño metodológico que contenía clases teórico-prácticas, actividades grupales y uso de plataformas. El curso complementario consistió en 13 sesiones y dos módulos: i) módulo I: orientado desde la psicología educacional y ii) módulo II: orientado al área de la enseñanza de la física. En la tabla 3 se presenta un resumen de los principales temas expuestos y trabajados por los estudiantes. En cada sesión de clases se realizaban actividades prácticas relacionadas con los contenidos de la tabla 3. Las estrategias de enseñanza más utilizadas fueron el ABP, actividades prácticas y estudios de casos. Diversos autores mencionan que éstas estrategias han mostrado resultados positivos en el proceso de enseñanza y aprendizaje de estudiantes universitarios (Faúndez et al., 2014; Ávila et al., 2016; Fierro et al., 2016; Cobos-Sanchiz et al., 2016; Bravo et al., 2016, Lee et al., 2017; Cobos et al., 2018). 
Tabla 3: Módulos y contenidos expuestos en el complementario.

\begin{tabular}{|l|l|c|}
\hline \multicolumn{1}{|c|}{ Módulos } & \multicolumn{1}{|c|}{ Contenidos } & Numero de clases \\
\hline \multirow{3}{*}{ Módulo I: Psicología Educativa } & $\begin{array}{l}\text { Fundamentos de la comunicación efectiva. } \\
\text { Aprendizaje significativo. } \\
\text { Aprendizaje Cooperativo. }\end{array}$ & 4 \\
\hline \multirow{3}{*}{ Módulo II: Enseñanza de la Física } & $\begin{array}{l}\text { Estrategias de resolución de problemas en física. } \\
\text { Aplicación de Entornos Virtuales de Aprendizaje (EVA). } \\
\end{array}$ & $\begin{array}{l}\text { Uso del Aprendizaje basado en problemas (ABP). } \\
\text { Estudio de Casos. } \\
\text { Uso de laboratorios experimentales básicos. }\end{array}$ \\
\hline
\end{tabular}

La tercera etapa se relaciona con la medición de la efectividad del curso. Los futuros tutores fueron grabados en la simulación de la realización de una ayudantía previa y posterior al curso, con el fin de evaluar los conocimientos, habilidades y actitudes desarrolladas. Para evaluar competencias específicas se utilizaron instrumentos evaluativos para actividades de proceso tales como pautas de observación y rúbricas, por parte de los docentes y los pares, desarrolladas en el transcurso de la asignatura. La encuesta de satisfacción cuenta con un apartado tipo likert con 15 ítems agrupados en tres dimensiones: cumplimento de objetivos de la asignatura, evaluación docente e implementación de la asignatura. Cuatro de los ítems no se encuentran agrupados en una dimensión ya que evalúan elementos específicos complementarios. En cada ítem los estudiantes podían puntuar su grado de acuerdo de 1 a 7 , siendo el valor 7 el máximo grado de acuerdo y 1 el máximo grado de desacuerdo. Además la encuesta comprende un componente cualitativo en la cual se les solicitaba a los estudiantes que señalaron fortalezas y aspectos a mejorar de la asignatura cursada. Tanto los instrumentos de evaluación como la encuesta de satisfacción fueron desarrollados por los docentes responsables de la asignatura, verificados por pares expertos de la misma casa de estudios, con el objetivo específico de evaluar este curso complementario.

\section{RESULTADOS}

Para medir el rendimiento del curso se utilizaron distintos instrumentos de evaluación como rubricas, pautas de observación y escalas de valoración, de modo tal que se obtuviera una calificación por estudiante. Podemos indicar que en el transcurso del tiempo los estudiantes han aprobado la asignatura con calificaciones sobresalientes. La tabla 4 muestra el promedio de calificaciones de estudiantes aprobados y reprobados.

Tabla 4: Resultados calificaciones año 2015, 2016 y 2017.

\begin{tabular}{|c|c|c|}
\hline Año & $N^{\circ}$ estudiantes & Promedio final \\
\hline 2015 & 8 & 5,3 \\
\hline 2016 & 5 & 6,8 \\
\hline 2017 & 35 & 6,3 \\
\hline
\end{tabular}

Los resultados de las calificaciones corresponden a: i) 3 evaluaciones del módulo de psicología educativa correspondiente a un $15 \%$ de la nota final, ii) 6 evaluaciones del módulo de didáctica correspondiente al $45 \%$ y iii) evaluación final correspondiente a la grabación de una ayudantía que pondera el $40 \%$ de la nota final. Al finalizar el curso se aplicó una encuesta de satisfacción de la asignatura, la cual fue respondida por $69 \%$ de los estudiantes $(n=33)$, la cual arrojó resultados positivos de los estudiantes con la asignatura en sus diversos elementos evaluados (Ver Tabla 5). Esta encuesta contaba con un ítem de preguntas abiertas, de la cuales se puede evidenciar respuestas favorables que demuestran la utilidad que presta para los estudiantes tanto en su desarrollo educativo como personal. Algunas respuestas se muestran en la tabla 6.

Tabla 5: Resultados encuesta de satisfacción por dimensión.

\begin{tabular}{|l|c|}
\hline \multicolumn{1}{|c|}{ Dimensión: Cumplimento de objetivos de la asignatura } \\
\hline La asignatura cumplió su objetivo de formar estudiantes como tutores/as. & 7,0 \\
\hline La asignatura me permitió adquirir conocimientos y habilidades para ser ayudante y tutor de asignaturas. & 6,7 \\
\hline $\begin{array}{l}\text { Las actividades realizadas en las clases me permitieron desarrollar conocimientos y habilidades de acuerdo } \\
\text { al objetivo del curso. }\end{array}$ & 6,7 \\
\hline Estoy satisfecho con los conocimientos y habilidades que adquirí en esta asignatura. & 6,7 \\
\hline \multicolumn{1}{|c|}{ Dimensión: Evaluación docente } & 7,0 \\
\hline Existía una buena disposición por parte de los docentes para responder consultas. & 7,0 \\
\hline El trato de los docentes fue cercano y respetuoso. & 7,0 \\
\hline La preparación y el dominio de contenidos de los docentes fueron adecuados. & \\
\hline
\end{tabular}


Tabla 5 (continuación)

\begin{tabular}{|c|c|}
\hline \multicolumn{2}{|l|}{ Dimensión: Implementación de la asignatura } \\
\hline La metodología utilizada en las clases fue adecuada para el cumplimiento de los objetivos. & 6,7 \\
\hline Los contenidos incluidos de la asignatura fueron pertinentes. & 6,8 \\
\hline El nivel de exigencia de las evaluaciones fue adecuado. & 6,7 \\
\hline Las evaluaciones realizadas en la asignatura me permitieron conocer los aspectos que necesitaba fortalecer. & 6,8 \\
\hline \multicolumn{2}{|l|}{ Otros elementos evaluados } \\
\hline La asignatura fue difundida ampliamente. & 6,1 \\
\hline El objetivo del complementario era conocido al inscribir la asignatura. & 7,0 \\
\hline La sala y el equipamiento utilizados para realizar las clases cumplían con los requerimientos de la asignatura. & 6,7 \\
\hline $\begin{array}{l}\text { Mi participación en las clases fue activa (consultas al profesor, realización de actividades grupales e } \\
\text { individuales). }\end{array}$ & 6,7 \\
\hline
\end{tabular}

Tabla 6: Respuestas de preguntas abiertas.

\begin{tabular}{|c|c|c|}
\hline Pregunta & \multicolumn{2}{|c|}{ Respuesta } \\
\hline $\begin{array}{l}\text { ¿Cuáles fueron los aspectos que } \\
\text { más te agradaron de la asignatura? }\end{array}$ & $\begin{array}{l}\text { Lo personalizado del curso } \\
\text { Que me ayudó a expresarme } \\
\text { Confío más en lo que puedo hacer } \\
\text { Es más fácil pararme frente a un } \\
\text { grupo } \\
\text { Me ayudó a mejorar mi letra y voz }\end{array}$ & $\begin{array}{l}\text { Me enseñaron a enseñar de forma } \\
\text { efectiva } \\
\text { Profesores muy buenos } \\
\text { Mejoré habilidades blandas } \\
\text { Cercanía y trato con los docentes }\end{array}$ \\
\hline $\begin{array}{l}\text { Para una próxima versión de la } \\
\text { asignatura, ¿Qué aspectos } \\
\text { mejorarías? }\end{array}$ & $\begin{array}{l}\text { Mayor propaganda } \\
\text { Que no sea tan largo } \\
\text { Las pautas de evaluación } \\
\text { Poder aplicar la ayudantía }\end{array}$ & $\begin{array}{l}\text { Mejorar la difusión de la asignatura } \\
\text { Subir la exigencia } \\
\text { Agregar más presentaciones orales. } \\
\text { Masificación de la asignatura }\end{array}$ \\
\hline
\end{tabular}

\section{CONCLUSIONES}

El curso de preparación de tutores es una alternativa que contribuye en la solución de deserción universitaria al fomentar la preparación de los estudiantes que realizan ayudantias en el área de física, por lo cual consideramos necesaria e importante la preparación de estos estudiantes de una manera integral, abarcando su preparación pedagógica desde una perspectiva constructivista. Este curso evidencia un cambio en el antes y después de los futuros tutores, puesto que en base a las grabaciones analizadas en conjunto por los docentes de la asignatura, se evidencia cómo sus estrategias en la entrega de contenidos, su desplante y seguridad cambió de forma positiva, presentando además un mejor orden y claridad en los problemas resueltos en la pizarra.

El carácter positivo de la asignatura se evidencia también en la evaluación de los propios estudiantes participantes, quienes consideran que la asignatura les permitió desarrollar las competencias para realizar labores formativas en el área de la física de una manera más planificada y clara. La implementación de la asignatura, a través de las metodologías utilizadas, se considera una fortaleza por parte de los docentes y estudiantes como elemento favorecedor de la adquisición de las competencias esperadas. Como elementos a mejorar, en base a la percepción de los propios estudiantes, se considera el aumento de las presentaciones orales por parte de los estudiantes para favorecer su práctica. En próximos estudios se fortalecerán las estrategias de evaluación de la asignatura para obtener resultados más robustos y articular la asignatura evaluando los posteriores procesos formativos liderados por los estudiantes participantes en ayudantías y/o acompañamientos tutoriales.

A pesar de que no se ha realizado una evaluación de desempeño en posteriores labores docentes de los estudiantes que han cursado la asignatura, la mayoría de ellos se encuentra realizando ayudantías en cursos de física o como tutores de la institución. Por lo tanto, como desafío en esta implementación, queda la evaluación de desempeño in situ de nuestros tutores, así como también incrementar la difusión de este curso de preparación e incentivar a mejorar las prácticas desde los inicios de la docencia.

\section{MATERIAL SUPLEMENTARIO}

Se dispone de material adicional que incluyen las pautas de evaluación y encuestas de satisfacción mencionadas en este trabajo. Se puede solicitar el material al autor principal. 


\section{REFERENCIAS}

Aguilar, W., G. Chávez y M. Fuentes, Tutorías: Estudio exploratorio sobre la opinión de los estudiantes de tronco común de ciencias de la ingeniería, Formación Universitaria, 10 (3), 69-80 (2017)

Álvarez, P. y M. González, La tutoría académica en la enseñanza superior: una estrategia docente ante el nuevo reto de la Convergencia Europea, Revista Electrónica Interuniversitaria de Formación del Profesorado, 19, 4-8 (2005)

Arbizu, F., C. Lobato y L. Del Castillo, Algunos modelos de abordaje de la tutoría universitaria, Revista de Psicodidáctica, 10 (2), 7-22 (2005)

Ávila, P., A. Elías, L. García y E. Camargo Zapata, Implementación de simulaciones virtuales en la enseñanza de física y química para la educación media en la subregión de Urabá, Antioquia, Tesis Doctoral, Escuela de ingenierías, Universidad Pontifica Bolivariana, Medellín, Colombia (2016)

Badiali, B. y N. Titus, Co-teaching: Enhancing student learning through mentor-intern partnerships, School-University Partnerships, 4(2), 74-80 (2010)

Baeten, M. y M. Simons, Student teachers' team teaching: Models, effects, and conditions for implementation, Teaching and Teacher Education, 41, 92-110 (2014)

Baeten, M., M. Simons, W. Schelfhout y R. Pinxten, Team teaching during field experiences in teacher education: Exploring the assistant teaching model, European Journal of Teacher Education, 1-21 (2018)

Baggini, I. y L. del Valle, Acción tutorial e integración universitaria, Congreso Clabes: Conferencia latinoamericana sobre abandono en la educación superior, Córdoba, Argentina 15-17 de noviembre (2017)

Bravo, A., G. Ramírez, C. Faúndez y H. Astudillo, Propuesta didáctica constructivista para la adquisición de aprendizajes significativos de conceptos en Física de fluidos, Formación Universitaria, 9 (2), 105-114 (2016)

Bulte, C., A. Betts, K. Garner y S. Durning, Student teaching: views of student near-peer teachers and learners, Medical teacher, 29(6), 583-590 (2009)

Cabrera-Murcia, P., ¿Cómo diseñar ayudantías que favorezcan el aprendizaje activo de los estudiantes tutoreados? La percepción del ayudante universitario, Estudios Pedagógicos, 43(3), 47-62 (2018)

Canales, A. y D. De Los Ríos, Retención de estudiantes vulnerables en la educación universitaria, Revista Calidad de la Educación, 30, 50-83 (2009)

Canales, A. y D. De los Ríos, Factores explicativos de la deserción universitaria, Calidad en la Educación, (26), 173201 (2018)

Cobos, H., P. Pérez y otros cuatro autores, Alumnos tutores y el aprendizaje de la lectura crítica en internado de pregrado, Investigación en educación médica, 7(26), 82-91(2018)

Cobos-Sanchiz, D., E. López-Meneses y M. Llorent-Vaquero, Propuesta de Innovación Didáctica con Portafolios Electrónicos en Formato Blog: Un Caso de Estudio en una Universidad, Formación Universitaria, 9(6), 27-42 (2016)

Colvin, J., Peer tutoring and social dynamics in higher education, Mentoring y Tutoring, 15(2), 165-181 (2007)

Corona, J., C. Jiménez y otros cuatro autores, Taller para formación de instructores de Introducción a la Cirugía, Investigación en Educación Médica, 7(26), 71-81 (2018)

Espejo, R. y J. González, El desarrollo de Profesores Universitarios y Escolares en Chile: ¿Brecha o Continuidad? Pontificia Universidad Católica de Valparaíso, Perspectiva Educacional, Formación de Profesores, 53 (2), 3-19 (2014)

Falchikov, N., Learning together. Peer tutoring in higher education, New York, Routledg (2001)

Faúndez, C., A. Bravo, A. Melo y H. Astudillo, Laboratorio Virtual para la Unidad Tierra y Universo como Parte de la Formación Universitaria de Docentes de Ciencias, Formación Universitaria, 7 (3), 33-40 (2014)

Fierro, E., C. Faúndez y J. Valderrama, Método de Contribución de Grupos: una Herramienta Fundamental en cursos Avanzados de Termodinámica y Física de Fluidos para la Estimación de Propiedades de Sustancias, Formación Universitaria, 9 (3), 99-108 (2016)

Filtz, T. y R. Gurung, Student perceptions of undergraduate teaching assistants, Teaching of Psychology, 40(1), 48-51 (2012)

Gairín J., F. Feixas, C. Guillamón y D. Quinquer, La tutoría académica en el escenario europeo de la Educación Superior, Revista interuniversitaria de formación del profesorado, (49), 61-78 (2004)

García, N., I. Asensio, R. Carballo, M. García y S. Guardia, La tutoría universitaria ante el proceso de armonización europea, Revista de Educación, 337, 189-210 (2005)

García, R., O. Cuevas, J. Vales e I. Cruz, Impacto de la tutoría presencial y virtual en el desempeño académico de alumnos universitarios, Revista Iberoamericana de Educación, 58(2), 1-11 (2012)

Garrido, C., La educación desde la teoría del capital humano y el otro, Educere, 11(36), 73-80 (2007)

Graffigna, A., L. Hidalgo y otros cuatro autores, Tutorial Practice as a Strategy of Retention at the School of Engineering, Procedia - Social and Behavioral Sciences, 116, 2489-2493 (2014) 
Lee, H., M. Longhurst y T. Campbell, Teacher learning in technology professional development and its impact on student achievement in science, International Journal of Science Education, 39(10), 1282-1303 (2017)

McCavit, K. y N. Zellner, Persistence of physics and engineering students via peer mentoring, active learning, and intentional advising, European Journal of Physics, 37(065702) 1-9 (2016)

Menéndez, J., El problema terminológico de la tutoría entre iguales y la afirmación de su especificidad didáctica, Observar, (4), 66-94 (2010)

Monereo, C., La investigación en la formación del profesorado universitario: hacia una perspectiva integradora, DOI:10.1174/021037013807533052, Infancia y Aprendizaje, 36 (3), 281-291 (2013)

Narro, J. y M. Arredondo, La tutoría: un proceso fundamental en la formación de los estudiantes universitarios, Perfiles educativos, 35 (141), 132- 151 (2013)

OCDE. Estudios de la OCDE: Educación superior en el desarrollo regional y de ciudades, https://www.oecd.org/edu/imhe/42945795.pdf (2009)

Pérez, C., I. Francés y M. Baeza, La percepción del alumnado sobre las competencias docentes del profesorado de la rama de Ciencias Sociales y Jurídicas de la Universitat de València, Teoría de la Educación; Educación y Cultura en la Sociedad de la Información, 14(3), 259 (2013)

Philipp, S., T. Tretter y C. Rich, Development of Undergraduate Teaching Assistants as Effective Instructors in STEM Courses, Journal of College Science Teaching, 45 (3), 74-82 (2016a)

Philipp, S., T. Tretter y C. Rich, Undergraduate Teaching assistant impact on student academic achievement, Electronic Journal of Science Education, 20 (2), 1-13 (2016b)

Rumbo, B. y T. Gómez, La acción tutorial en un contexto universitario masificado y la reivindicación europea de su valor formativo, Revista de Formación e Innovación Educativa Universitaria, 4(1), 13-34 (2011)

Sánchez, M. y C. Mayor, Los jóvenes profesores universitarios y su formación pedagógica, Claves y controversias, Revista de Educación, (339), 923-946 (2006)

SIES. Panorama de la Educación Superior en Chile 2014. División de Educación Superior. Ministerio de Educación (2014)

Solaguren-Beascoa, M. y L. Moreno, Escala de actitudes de los estudiantes universitarios hacia las tutorías académicas, Educación, 21, 19(1) (2016)

Sommariva, C., A. Apip, C. Fernández y R. Motzfeld, Evaluación del Programa Colaborador Académico-Alumno, en la Asignatura de Operatoria Dental, Facultad de Odontología, Universidad de Chile, Revista de Educación en Ciencias de la Salud, 10(1), 23-28 (2013)

Sospedra, M., M. Lloret y T. Cañas, Percepción de los estudiantes de ingeniería civil sobre las competencias óptimas del docente universitario, Sinéctica, (41), 2-20 (2013)

Tinto, V., From theory to action: Exploring the institutional conditions for student retention. J.C. Smart (Ed.), Higher education: Handbook of theory and research (2010)

Vega G., Á. Ferrat y C. Busoch, Una Experiencia Educativa: Un Programa De Mentoría Estudiantil En Ingeniería Automática De La Cujae. Congreso Clabes: Conferencia latinoamericana sobre abandono en la educación superior, Talca, Chile 11,12 y 13 de Noviembre (2015)

Vygotsky, L., El desarrollo de los procesos psicológicos superiores, Barcelona, Editorial Crítica (1979)

Yin, R., Case Study Research. Design and Methods, London, SAGE (1989) 
